\title{
Relationship Between Optical Coherence Tomography Angiography Vessel Density and Severity of Visual Field Loss in Glaucoma
}

\author{
Adeleh Yarmohammadi, MD¹, Linda M. Zangwill, PhD'1, Alberto Diniz-Filho, MD, PhD', Min \\ Hee Suh, MD1, Siamak Yousefi, PhD1', Luke J. Saunders, PhD1, Akram Belghith, PhD1, \\ Patricia Isabel Manalastas, MD ${ }^{1}$, Felipe A. Medeiros, MD, PhD ${ }^{1}$, and Robert N. Weinreb, MD ${ }^{1}$ \\ ${ }^{1}$ Hamilton Glaucoma Center, Shiley Eye Institute, Department of Ophthalmology, University of \\ California San Diego, La Jolla, CA.
}

\section{Abstract}

Purpose-To evaluate the association between vessel density measurements using optical coherence tomography angiography (OCT-A) and severity of visual field loss in primary openangle glaucoma (POAG)

Design-Observational cross-sectional study

Participants-One hundred and fifty three eyes from 31 healthy, 48 glaucoma suspects, and 74 glaucoma participants enrolled in the Diagnostic Innovations in Glaucoma Study

\begin{abstract}
Methods-All eyes underwent imaging using an OCT-A (Angiovue, Optovue; Fremont, CA) and a spectral domain OCT (Avanti, Optovue; Fremont, CA), along with standard automated perimetry (SAP). Retinal vasculature information was summarized as vessel density, the percent of area occupied by flowing blood vessels in the selected region. Two measurements from the retinal nerve fiber layer (RNFL) were utilized: circumpapillary vessel density (cpVD) $(750-\mu \mathrm{m}$-wide elliptical annulus around the optic disc); and whole image vessel density (wiVD) (entire $4.5 \times 4.5$ mm scan field)
\end{abstract}

\footnotetext{
Correspondence: Robert N. Weinreb, MD, Hamilton Glaucoma Center and Department of Ophthalmology, University of California, San Diego, 9500 Gilman Drive, La Jolla, CA, 92093-0946, rweinreb@ucsd.edu.

Publisher's Disclaimer: This is a PDF file of an unedited manuscript that has been accepted for publication. As a service to our customers we are providing this early version of the manuscript. The manuscript will undergo copyediting, typesetting, and review of the resulting proof before it is published in its final citable form. Please note that during the production process errors may be discovered which could affect the content, and all legal disclaimers that apply to the journal pertain.

Financial Disclosure(s): Adeleh Yarmohammadi: none; Linda M. Zangwill: Research support - Carl Zeiss Meditec, Heidelberg Engineering, National Eye Institute, Topcon, and Nidek; Alberto Diniz-Filho: none; Min Hee Suh: none; Siamak Yousefi: none; Luke J. Saunders: none; Akram Belghith: none; Patricia Isabel Manalastas: none; Felipe A. Medeiros: Financial support - Alcon, Allergan, Bausch \& Lomb, Carl Zeiss Meditec, Heidelberg Engineering, Merck, Reichert, Sensimed, and Topcon; Research support - Alcon, Allergan, Carl Zeiss Meditec, National Eye Institute; and Reichert; Consultant - Allergan, Carl Zeiss Meditec, and Novartis; David Huang: Financial support - Optovue; Consultant - Carl Zeiss Meditec, Optovue; Robert N. Weinreb: Research support -Carl Zeiss Meditec, Genentech, Heidelberg Engineering, National Eye Institute, Optovue, and Topcon; Consultant - Alcon, Allergan, Bausch \& Lomb, ForSight, Unity

Vessel density measurements obtained by optical coherence tomography angiography (OCT-A) are significantly associated with severity of glaucomatous visual field damage independent of neural structural loss. OCT-A vascular measures have promise for enhancing our understanding the pathophysiology and management of glaucoma.
} 
Main Outcome Measures-Associations between severity of visual field loss, reported as SAP mean deviation (MD) and OCT-A vessel density

Results-Compared to POAG eyes, normal eyes demonstrated a denser microvascular network within the RNFL. Vessel density was higher in normal eyes followed by glaucoma suspects, mild glaucoma and moderate to severe glaucoma eyes for wiVD (55.5, 51.3, 48.3, 41.7\% respectively) and for cpVD $(62.8,61.0,57.5,49.6 \%$ respectively) ( $\mathrm{P}<0.001$ for both). The association between the severity of visual field damage (MD) with cpVD and wiVD was stronger $\left(\mathrm{R}^{2}=0.54\right.$, and $R^{2}=0.51$ respectively) than the association between visual field MD and $R N F L\left(R^{2}=0.36\right)$ and rim area $\left(\mathrm{R}^{2}=0.19\right)(\mathrm{P}<0.05$ for all). Multivariate regression analysis, adjusted for confounders, showed that each $1 \%$ decrease in cpVD was associated with $0.64 \mathrm{~dB}$ loss in MD and each $1 \%$ decrease in wiVD, was associated with $0.66 \mathrm{~dB}$ loss in MD. In addition, the association between vessel density and the severity of visual field damage was found to be significant even after controlling for the effect of structural loss

Conclusions-Decreased vessel density was significantly associated with severity of visual field damage independent of the structural loss. OCT-A is a promising technology in glaucoma management, potentially enhancing the understanding of vascular role in the pathophysiology of the disease

\section{INTRODUCTION}

Glaucoma is a progressive optic neuropathy with unknown etiology characterized by degeneration of retinal ganglion cells (RGC) and their axons resulting in a characteristic appearance of the optic disc and visual field loss. ${ }^{1}$ There is increasing evidence that optic nerve blood flow impairment and microcirculatory deficiency may have a role in the pathogenesis of glaucoma. ${ }^{2-4}$ Although the details of this relationship have not been established precisely ${ }^{5-7}$ This is in part due to the instrumentation that has been available and their difficulty of accurately measuring ocular blood flow. 8,9

In contrast to ocular blood flow, objective, accurate, and quantitative measurements of the optic nerve head and macula can be obtained with optical coherence tomography (OCT), and they have become the standard for structural evaluation in glaucoma research and clinical practice. However, structural measurements have only moderate correlation with visual field loss. ${ }^{10-12}$

It recently has become possible to obtain non-invasive images to characterize retinal vasculature with OCT angiography (OCT-A) ${ }^{13}, 14$. OCT-A provides reproducible quantitative assessment of the microvasculature in the optic nerve head, peripapillary retina, and macula. ${ }^{15-20}$ Recent studies using OCT-A have suggested that this new technology might be useful in the diagnosis, staging, and monitoring of glaucoma. ${ }^{16,18-20}$ These measurement also may clarify the role of microcirculation and optic nerve blood flow in the pathogenesis of glaucoma.

The current study evaluates the relationship between OCT-A retinal vessel density parameters with functional measurements and compare it to standard spectral domain OCT (SD-OCT) structural measurements. 


\section{METHODS}

This was an observational cross-sectional study including 153 eyes from 31 healthy, 48 glaucoma suspect, and 74 primary open angle glaucoma (POAG) patients enrolled in the Diagnostic Innovations in Glaucoma Study (DIGS) who underwent OCT-A (Angiovue; Optovue Inc. Fremont, CA, USA), ${ }^{13-20}$ and SD-OCT optic nerve head imaging (Avanti; Optovue Inc., Fremont, CA, USA).

The DIGS eligibility criteria and methodological details have been reported in previous studies. ${ }^{21}$ In brief, all participants completed a comprehensive ophthalmologic examination, including best corrected visual acuity (BCVA), slit-lamp biomicroscopy, intraocular pressure (IOP) measurement with Goldmann applanation tonometry, gonioscopy, dilated fundus examination, stereoscopic optic disc photography, ultrasound pachymetry, and standard automated perimetry (SAP) in both eyes. Only participants over 18 years of age with open angles on gonioscopy, and spherical refraction within $\pm 10 \mathrm{D}$ were included.

Written informed consent was obtained from all participants. The Institutional Review Board at the University of California San Diego approved all protocols and methods described were in agreement with the tenets of the Declaration of Helsinki and the Health Insurance Portability and Accountability Act (HIPAA).

Healthy subjects were required to have an IOP of $21 \mathrm{mmHg}$ or less with no history of elevated IOP, normal appearing optic discs, intact neuroretinal rims and retinal nerve fiber layer (RNFL), and normal visual field test results defined as a Pattern Standard Deviation (PSD) within the 95\% confidence limits, and Glaucoma Hemifield Test (GHT) result within normal limits. Glaucoma suspects had either an IOP $\geq 22 \mathrm{mmHg}$ and/or suspicious appearing optic discs without evidence of repeatable glaucomatous visual field damage.

Glaucoma was defined by the presence of repeatable abnormal SAP results with a GHT outside normal limits or PSD outside the $95 \%$ normal limits. Glaucoma patients were additionally classified into 2 groups based on the severity of their visual field damage; mild glaucoma was defined as visual field mean deviation (MD) higher than $-6 \mathrm{~dB}$ and moderate to severe glaucoma as a visual field MD lower than $-6 \mathrm{~dB} .{ }^{22}$ To ensure comparability of age across study groups, only subjects $\geq 45$ years were included.

Eyes with history of intraocular surgery (except for glaucoma surgery or uncomplicated cataract surgery), secondary causes of glaucoma, non-glaucomatous optic neuropathies, vascular or non-vascular retinopathies, and other ocular or systemic diseases known to impair the visual field were excluded from the investigation.

Two blood pressure (BP) measurements obtained in a resting, seated position were taken at least 5 minutes apart using an Omron Automatic (Model BP791IT; Omron Healthcare, Inc., Lake Forest, IL, USA) instrument. Mean arterial pressure (MAP) was calculated as MAP= $1 / 3$ systolic $\mathrm{BP}+2 / 3$ diastolic $\mathrm{BP}$ and mean ocular perfusion pressure (MOPP) was defined using the following equation: $\mathrm{MOPP}=2 / 3 \mathrm{MAP}-\mathrm{IOP}$. 


\section{Standard Automated Perimetry (SAP)}

All participants underwent visual field testing using 24-2 pattern Swedish interactive threshold algorithm on the Humphrey Field Analyzer (Carl Zeiss Meditec, Dublin, CA, USA) within 6 months of imaging. Only reliable tests ( $₫ 3 \%$ fixation losses and false negatives, and $₫ 5 \%$ false positives) were included. The quality of visual field tests was also reviewed by the Visual Field Assessment Center (VisFACT) ${ }^{23}$ staff to identify and exclude visual fields with evidence of inattention or inappropriate fixation, artifacts such as eyelid and lens rim artifacts, fatigue effects and abnormal results caused by diseases other than glaucoma.

\section{Optical Coherence Tomography Angiography}

The OCT-A imaging system provides a noninvasive method for visualizing the optic nerve head and retinal vasculature. The image acquisition technique is optimized for the SplitSpectrum Amplitude-Decorrelation Angiography (SSADA) algorithm described in detail elsewhere. ${ }^{13}$ The SSADA method captures the dynamic motion of moving scatters such as red blood cells in a flowing blood vessel and computes a high-resolution three-dimensional (3D) visualization of perfused vasculature.

The OCT-A characterizes vascular information at each retinal layer as an en face angiogram, a vessel density map (Figure 1), and quantitatively as vessel density (\%), calculated as the percentage area occupied by flowing blood vessels in the selected region.

For this study, we utilized vessel density measurements within the peripapillary RNFL in scans with a $4.5 \times 4.5 \mathrm{~mm}$ field of view centered on the optic nerve head. Vessel density within the RNFL was measured from the internal limiting membrane (ILM) to RNFL posterior boundary using standard AngioVue software (version 2015.1.0.90). Measurements were calculated in two areas. Whole image vessel density (wiVD) was obtained over the entire $4.5 \times 4.5 \mathrm{~mm}$ scan field, and circumpapillary vessel density (cpVD) was measured in a $750-\mu \mathrm{m}$-wide elliptical annulus extending outward from the optic disc boundary, where the inner elliptical contour is obtained by fitting an ellipse to the disc margin on the OCT en face retinal angiogram and the ring width between inner and outer elliptical contour is defined as the circumpapillary region (Figure 1).

The Imaging Data Evaluation and Analysis (IDEA) Reading Center established a standard protocol for OCT-A image quality review. Trained graders reviewed all images to identify poor quality scans, defined as blurred images, scans with an SSI of less than 48, residual motion artifacts visible as irregular vascular pattern or disc boundary on the enface angiogram, local weak signal caused by floaters and RNFL segmentation errors. Graders also reviewed the location of the optic disc margin for accuracy and if needed the margin was adjusted manually and confirmed by two graders.

\section{Spectral Domain Optical Coherence Tomography}

Avanti SD OCT uses an 840-nm central wavelength, a 22- $\mu \mathrm{m}$ focal spot diameter, and 70$\mathrm{kHz}$ axial line scan rate that yields an axial resolution of 5- $\mu \mathrm{m}$ in tissue. The optic nerve head (ONH) map image acquisition protocol was used to obtain RNFL thickness 
measurements in a 10 pixel-wide band along a $3.45 \mathrm{~mm}$ diameter circle centered on the $\mathrm{ONH}$ and rim area measurements.

All participants had both SD-OCT and OCT-A imaging performed on the same day. Participants having poor quality ONH scans defined by an SSI of lower than 37 and scans with segmentation failure or artifacts were excluded from the analysis.

Three hundred fifty-one eyes of 213 subjects had OCT-A and SD-OCT imaging within 12 months of visual field testing and were potentially eligible for inclusion in the analysis. Fifty-four eyes were excluded due to poor quality OCT-A scans, 16 eyes were excluded due to poor quality of SD-OCT images, and 35 eyes were excluded due to unreliable visual field tests. 246 eyes of 153 subjects had good quality OCT-A, SD-OCT and visual field tests. One from these 153 subjects was randomly selected to be included in the analysis.

\section{Statistical Analysis}

One eye of each subject was randomly selected to be included in the analyses. Descriptive statistics were calculated as the mean and standard deviation (SD) and categorical variables were compared using the chi-square test. Analysis of variance (ANOVA) was performed to compare mean values among the healthy, glaucoma suspect, mild glaucoma and severe to moderate glaucoma eyes.

Relationships between visual field parameters and OCT-A vessel density and SD-OCT RNFL and rim area were evaluated using simple linear $(y=a x+b)$ and second-order polynomial (or quadratic) models $\left(y=a x^{2}+b x+c\right)$. Results were reported as $\mathrm{R}^{2}$ (coefficient of determination) with differences between the $\mathrm{R}^{2} \mathrm{~s}$ calculated using bootstrapping procedures to estimate the $95 \%$ confidence intervals (CI) of the difference in coefficients of determination. Akaike's information criterion (AIC) was used to compare the models for goodness of fit, ${ }^{24}$ The smaller the AIC, the better the model. Univariable linear regression models were built using visual field $\mathrm{MD}$ as the dependent variable and OCT-A parameters, wiVD and cpVD and SD-OCT RNFL thickness and rim area measurements and other and demographic ocular characteristic variables as the independent variables.

Multivariable models were also used to evaluate the relationship between the visual field MD with vessel density and SD-OCT RNFL and rim area while adjusting for potential confounding parameters such as age, IOP, central corneal thickness (CCT) and axial length.

All statistical analyses were performed with Stata version 14 (StataCorp, College Station, TX), and JMP version 11.2.0 (SAS Inc., Cary, NC). The alpha level (type I error) was set at 0.05 for all comparisons.

\section{RESULTS}

The study population consisted of 31 healthy subjects (mean age 69.0 \pm 7.7 years, SAP MD $0.3 \pm 1.3 \mathrm{~dB}$ ), 48 glaucoma suspects (mean age $71.4 \pm 9.4$ years, SAP MD $-0.6 \pm 1.5 \mathrm{~dB}$ ), 46 mild glaucoma patients (mean age $72.9 \pm 10.7$ years, SAP MD $-3.0 \pm 1.8 \mathrm{~dB}$ ) and 28 moderate to severe glaucoma patients (mean age $75.7 \pm 10.7$ years, SAP MD $-13.6 \pm 6.6$ 
dB). (Table 1). Healthy subjects tended to be younger than glaucoma suspects and glaucoma patients, but this difference was not statistically significant (ANOVA $\mathrm{P}=0.063$ ).

Qualitative assessment: Healthy eyes generally appeared to have denser capillary networks in the RNFL layer compared to eyes with early glaucomatous optic nerve damage and trend of a sparser microvascular network could be detected with advancing stages of the disease (Figure 1).

Quantitative assessment: Vessel density measurements were lower in more severe disease. Specifically, the mean wiVD in moderate to severe glaucoma eyes was significantly lower $(41.7 \pm 5.5 \%$, ) than in mild glaucomatous eyes $(48.3 \pm 4.2 \%)$, glaucoma suspects $(51.3$ $\pm 4.6 \%$ ) and healthy eyes $(55.5 \pm 3.2 \%$ ) (ANOVA $\mathrm{P}<0.001$, Tukey HSD $\mathrm{P}<0.05$ for all comparisons). (Table 1) Mean cpVD values were also significantly lower in moderate to severe glaucoma eyes $(49.6 \pm 6.9 \%$,) followed by mild glaucoma $(57.5 \pm 4.4)$, glaucoma suspects $(61.0 \pm 4.7 \%)$ and healthy eyes $(62.8 \pm 3.9 \%)$, (ANOVA P $<0.001$, Tukey HSD $\mathrm{P}<0.05$ for all pairwise comparison except between healthy and glaucoma suspect eyes. $(\mathrm{P}=0.322)$. Standard structural and functional measurements also showed statistically significant differences among groups $(\mathrm{P}<0.001$, Table 1).

Results of age-adjusted univariate linear regressions are summarized in Table 2. Linear and curvilinear (quadratic) relationships between OCT-A vessel density and SD-OCT structural measurements with visual field MD and visual field PSD are illustrated in Figures 3 and 4, respectively. The strength of the associations between structure (expressed in linear scales) and different visual field indices (expressed in both $\mathrm{dB}$ and linear scales) using both linear and quadratic regression models are summarized in Table 3. We used AIC to compare the linear and curvilinear regression models of OCT-A vessel density, and SD-OCT RNFL thickness and rim area with visual field MD, PSD, mean sensitivity (dB) and mean sensitivity in $1 /$ lambert (Table 3 ). The quadratic model was better than the linear model in assessing the relationship between visual field measurements and vessel density parameters, as well as between visual field measurements and structural measurements (Table 3).

Significant differences were found comparing strength of the associations between MD and both OCT-A vascular parameters with the association between MD and RNFL and rim area measurements ( $\mathrm{P} \unlhd 0.05$ for all pairwise comparisons). The association between $\mathrm{MD}$ and RNFL thickness was also significantly stronger than between MD and rim area $(\mathrm{P}=0.001)$. The associations between wiVD and cpVD with MD were similar $(\mathrm{P}=0.500)$. The strongest associations with visual field PSD were with wiVD, $\mathrm{cpVD}\left(\mathrm{R}^{2}=0.39\right.$ and 0.36 respectively), and RNFL $\left(R^{2}=0.37\right)$ followed by rim area $\left(R^{2}=0.23\right)$. Significant differences were found between the associations of PSD with wiVD and rim area $(\mathrm{P}=0.026)$ and between RNFL thickness and rim area $(\mathrm{P}=0.035)$. The linear associations between visual field mean sensitivity were strongest with cpVD $\left(\mathrm{R}^{2}=0.55\right)$ followed by wiVD $\left(\mathrm{R}^{2}=0.53\right), \mathrm{RNFL}$ thickness $\left(\mathrm{R}^{2}=0.37\right)$ and rim area $\left(\mathrm{R}^{2}=0.19\right)$. After converting mean sensitivity from logarithmic $(\mathrm{dB})$ to linear units (1/lambert), a similar pattern was found; Association with mean sensitivity ( $1 /$ lambert), were highest for wiVD and $\operatorname{cpVD}\left(\mathrm{R}^{2}=0.44\right.$ for both) followed by $R N F L$ thickness $\left(R^{2}=0.34\right)$ and rim area $\left(R^{2}=0.18\right)$. 
The strength of the associations between visual field MD with structural and OCT-A measures also were compared using a curvilinear quadratic model. The associations between OCT-A and visual field MD were significantly stronger than the associations between visual field MD and RNFL and rim area $(\mathrm{P}<0.05$ for all pairwise comparisons using bootstrapping procedure).

Results from univariate regression analysis for visual field MD as the dependent variable are summarized in Table 4. Multivariate linear regression analysis, while controlling for the potentially confounding effect of age, IOP, CCT, and axial length, showed that each $1 \%$ decrease in cpVD, was associated with $0.64 \mathrm{~dB}$ loss in $\mathrm{MD}(\mathrm{P}<0.001)$, and each $1 \%$ decrease in wiVD, was associated with $0.66 \mathrm{~dB}$ loss in $\mathrm{MD}(\mathrm{P}<0.001)$.

Multivariate regression analysis that controlled for the effect of potential confounders (age, IOP, CCT and axial length) and also adjusted for the effect of RNFL thickness (Table 5), showed that wiVD was independently associated with visual field MD. Similar results were found when cpVD was included in the model instead of wiVD. The association between RNFL with MD was no longer statistically significant when vessel density was included in the model. The multivariate regression analysis also was completed using rim area instead of RNFL thickness, and the results were similar; Each $1 \%$ decrease in wiVD, was associated with $0.71 \mathrm{~dB}$ loss in $\mathrm{MD}(\mathrm{P}<0.001)$ and the association between rim area and MD were no longer significant $(\mathrm{P}=0.285)$ when wiVD was included in the model.

For completeness, associations between clinical and ophthalmic features and OCTA vessel density also were evaluated. OCT-A vessel density was significantly associated with RNFL and rim area measurements ( $\mathrm{P}<0.001$, Table 2). As structural measurements, such as RNFL, optic nerve head rim and cup area have been shown to be associated with disc size, we also evaluated the association of ONH area on OCT-A vascular measurements. There were no statistically significant correlations between disc area with wiVD and cpVD measurements in healthy eyes $\left(\mathrm{R}^{2}=0.005, \mathrm{P}=0.696\right.$, and $\mathrm{R}^{2}=0.009, \mathrm{P}=0.614$, respectively). For this reason, disc area was not controlled for in the multivariable analyses. In addition, we did not find a significant association between MOPP and $\operatorname{cpVD}\left(\mathrm{R}^{2}=0.003, \mathrm{P}=0.49\right)$ or wiVD $\left(\mathrm{R}^{2}=0.000\right.$, $\mathrm{P}=0.85)$.

\section{DISCUSSION}

Results of the present study demonstrate a significant relationship between vessel density and severity of visual field damage. Qualitatively, the OCT-A vessel density map showed sparser peripapillary vascular networks in more severe glaucoma. Quantitatively, lower vessel density values, were associated with more advanced stages of glaucomatous visual field damage. The principal finding of the study was a relatively strong association between $\mathrm{cpVD}$, wiVD, and visual field loss expressed as $\mathrm{MD}\left(\mathrm{R}^{2}=0.54\right.$ and 0.51 , respectively, $\mathrm{P}<0.001$ for both) suggesting that reduced OCT-A vessel density is associated with more severe glaucoma. Our results also suggest the vascular-functional correlations were stronger than the standard structural (RNFL and rim area)-function relationships whether comparing linear or non-linear fitted models. Moreover, multivariate analyses indicated an independent 
relationship between reduced vessel density and visual field loss, even after adjusting for the severity of structural damage measured by rim area and RNFL thickness.

Findings of a relatively strong correlation between vessel density measurements and visual field loss are in accordance with previous reports using OCT-A vessel density. ${ }^{16}, 18,20$ However, it is worth mentioning that these reports measured vessel density in a thick retinal slab from internal limiting membrane (ILM) to retinal pigment epithelium (RPE) while our results focused on vessel density in a more superficial RNFL, from the ILM to RNFL posterior boundary. To our knowledge there has been no previous reports on the vascular network in the RNFL in glaucomatous eyes. Therefore, our study is unique in that we found that glaucoma eyes had significantly sparser vessel density in the RNFL layer compared to both glaucoma suspects and healthy eyes, and that these vessel density measurements were lowest in the glaucoma eyes with most severe disease.

In order to measure the vasculature within the RNFL, the boundaries were detected based on conventional retinal layer segmentation of the SD-OCT images. Glaucoma is characterized by re-modeling of the optic nerve head tissue. However, even in the presence of tissue remodeling, the RNFL boundaries can be reliably detected based on the backscattered intensity image from the SD-OCT. There is spatial co-localization laterally and in depth between the SD-OCT intensity and the OCT-A image.

Findings of decreased OCT-A vascular parameters in glaucoma patients are also in general agreement with the large body of evidence suggesting hemodynamic impairments in optic nerve head, retina, choroid, and retrobulbar circulations in glaucoma eyes. ${ }^{25-29}$ In addition, previous OCT-A reports compared the correlations between visual field loss and vessel density with the correlations between visual field loss and RNFL thickness. ${ }^{16,18}$ Consistent with our results, they reported stronger associations between visual field damage and vessel density. However, the current study went further and included two structural measures with vessel density in the multivariate model to demonstrate that vessel density was still strongly associated with visual field damage even after controlling for standard structural measures in the model. This finding is in agreement with Hwang et $\mathrm{al}^{7}$ who reported an independent relationship between visual field MD and another measure of retinal vascular integrity, namely total retinal blood flow measured by Doppler-OCT.

There are several possible explanations for the independent association of vessel density and visual field MD: first, it may be due, at least in part, to the existence of dysfunctional (i.e. pre-apoptotic) retinal ganglion cells (RGCs) that may have reduced blood flow and therefore lower vessel density as well as poorer visual field sensitivity. As these dysfunctional retinal ganglion cells have not yet atrophied, a reduction in RNFL thickness and rim area may not yet be detectable. Moreover, histologic studies also showed only moderate agreement between RNFL thinning and retinal ganglion cell loss, ${ }^{30,} 31$ suggesting that RNFL thinning does not completely reflect the functional status of retinal ganglion cells. Therefore, the stronger correlation between vessel density and visual field damage might suggest that vessel density is a better reflection of retinal ganglion cell functioning than structural loss. 
The relationship between detectable structural and functional damage and change in glaucoma is complex ${ }^{32}$ and our results suggest that the relationship between OCT-A vessel density and visual field measures is also complex and influenced by many factors. Previous reports on standard structure-function relationships suggested that the strength of these relationships depends on the methods and scales of visual field expression, the type of structural measuring device and the characteristics of the studied population. ${ }^{32}$ Therefore, several global visual field indices, including MD, PSD, and mean sensitivity were assessed and compared using both linear and curvilinear associations between SAP functional measurements and vessel density, RNFL thickness, and rim area.

As the nature of the association between new vascular parameters and functional measures is not well established, we also investigated whether the relationship between vessel density and visual field damage was linear or curvilinear. Previous reports on OCT-A demonstrated a linear relationship between vessel density and visual field measurements. ${ }^{16,18}$ Our findings, based on AIC analysis suggest that a quadratic model provides a somewhat better fit to the relationship between vessel density and visual function than a linear model.

However AIC values are only used for comparison between a set of candidate models and do not suggest the adequacy of the preferred model. ${ }^{24}$ Future studies are required to explore the exact nature of these relationships.

In our study, SAP MD, PSD and MS measured in a logarithmic scale reported in decibels and also MS converted to a linear scale reported in $1 /$ lambert were significantly associated with vessel density measurements ( $\mathrm{P}<0.001$ for all). The associations between $\mathrm{cpVD}$, wiVD and $\mathrm{MD}\left(\mathrm{R}^{2}=0.54\right.$ and 0.51 , respectively) were higher than their association with PSD $\left(\mathrm{R}^{2}=0.36\right.$ and 0.39 respectively, $\left.\mathrm{P}<0.001\right)$. Reports comparing the strength of the association between OCT based vascular measurements and different visual field summary measures are inconsistent. Hwang et $\mathrm{al}^{7}$ showed that total retinal blood flow measured by Doppler-OCT was highly correlated with MD, but its relationship with PSD did not reach statistical significance. Another Doppler-OCT based study ${ }^{33}$ investigating hemispheric retinal blood flow measurements in eyes having glaucomatous visual field damage confined to a single hemifield, reported significant differences in blood flow measurements between the affected and unaffected retinal hemispheres in glaucoma patients compared to healthy age-matched subjects, but failed to find an association between hemispheric retinal blood flow measurements and visual field mean retinal sensitivity measured as 1/lambert in the corresponding hemifield. In a recent OCT-A study, Liu et a ${ }^{18}$ reported that peripapillary vessel density measured in a circumpapillary ring was more strongly correlated with visual field PSD compared to MD. Finally, more consistent with our findings, Wang et al ${ }^{20}$ demonstrated that optic disc OCT-A vessel density correlated with both SAP MD and PSD, but was more strongly correlated with MD.

The conflicting results in recent OCT based Doppler and OCT-A studies investigating the relationship between vascular measurements and visual field abnormalities could be attributable, in large part, to different aspects of retinal vasculature that were measured. Moreover, the investigated study populations vary regarding patient's risk profile, the severity and pattern of glaucoma damage, and systemic factors that might have an effect on ocular hemodynamics. 
Reduced vessel density can be either the result of capillary dropout or of very low or absent flow. If vessel density is lower in more advanced glaucoma eyes, it would indicate one or both scenarios may be occurring. Vessel density measurement within the RNFL is not a direct quantification of the blood flow. But if flow in certain vascular structures is reduced to a level below the detection limit of OCT-A in diseased eyes while clearly detectable in normal eyes, it is possible that lower vessel density may be a surrogate indicator of decreased blood flow in the diseased eyes. Comparison of validated measures of blood flow such as Doppler-OCT to OCT-A vessel density in longitudinal studies may help answer this important question.

Although there is general consensus that ocular blood flow is reduced in glaucoma, 3,34 studies investigating this issue have been hampered by the lack of a reproducible method to measure aspects of ocular circulation. OCT is a widely available tool for structural assessment in glaucoma management that is now used extensively as standard clinical care. Using OCT-A technology for ocular hemodynamic evaluations not only offers the advantage of providing a quantitative assessment of ocular circulation at a level of precision that has not been achieved with previous instruments that measured blood flow, ${ }^{9,} 18$ but also its feasibility from a clinical standpoint suggests that OCT-A may be a useful modality which may reflect hemodynamic considerations relevant to glaucoma management. Moreover, the device's ability to visualize the vascular networks in easily interpretable images and density maps (Figure 1) may provide new clinical relevant information that can easily be incorporated into routine management of glaucoma patients.

It should be noted that the current OCT-A device detects vessels based on amplitude decorrelation, which results from blood flow however it does not directly quantify flow within these vessels. In fact, decorrelation is linearly related to blood flow only over a limited range above a certain threshold of motion. In other words, vessels with very slow or absent flow below the detection threshold of the instrument will not be detected and for the vessels detected, the current instrument does not differentiate a vessel with faster flow from one that has slower flow. ${ }^{35}$ For these reasons, the term vessel density is used as a quantitative summary measure of the vascular structures detected that reflects the proportion of area occupied by flowing vessels. Another known limitation of OCT-A technology is projection artifacts, which results from ghost images of anterior vessels projecting posteriorly. ${ }^{13,36}$ However, projection artifacts do not affect the measurements in the current study because microvasculature within the RNFL is the most anterior retinal vasculature and therefore not affected by projection artifact. Although results of the present study and previous reports using OCT-A, 15, 16, 18-20 suggest lower vessel density in glaucoma and glaucoma suspect eyes may be relevant to the pathophysiology of the disease, the concept of vessel density is not well understood. In an earlier study with a similar technology, OCTmicroangiography, Zhi et.al ${ }^{37}$ documented disappearance of signal from the peripapillary microvasculature in the rat. However, there was persistence of some signal in large vessels in this region despite high IOP $(100 \mathrm{mmHg})$. Comparison of in-vivo assessment of OCT-A vessel density to histopathological studies is needed to clarify what vessel density is measuring. 
The present study has several other limitations. We used visual field indices to reflect the severity of glaucoma. However, other non-glaucomatous factors such as refractive error, lenticular and media opacities ${ }^{38}$ may also contribute to the visual field MD, PSD and mean sensitivity. Moreover, our study population included few patients with advanced glaucoma. Recent reports suggest that standard structural measures such as RNFL thickness reach a floor effect and visual field tests are highly variable in eyes with advanced glaucoma. ${ }^{11,39-42}$ It is important to evaluate whether OCT-A has a sufficient dynamic range to provide clinically relevant information across the full spectrum of glaucoma severity. In addition, we did not evaluate the possible confounding impact of various systemic conditions, blood pressure and perfusion pressure, glaucoma eye drops, and systemic medications on vessel density and its relationship to standard structural and functional measures. It also should be noted that the cross-sectional design of the current study limits the determination of the temporal relationship between OCT-A vessel density loss and glaucomatous structural and functional damage. Longitudinal studies are necessary to evaluate the topographic and temporal relationship between changes in OCT-A vessel density and glaucomatous changes in standard structural and functional measures in healthy, glaucoma suspect, and glaucoma patients.

In conclusion, OCT-A vessel density measurements are significantly associated with severity of visual field damage. These associations are generally stronger than standard structural measures such as RNFL and rim area. Moreover, OCT-A vessel density measurements are still significantly associated with severity of visual field loss even after adjusting for standard structural measurements. For these reasons, OCT-A is a promising technology that will allow clinical monitoring of vascular changes in glaucoma, and it could potentially allow further understanding on the pathophysiology of the disease, specifically its underlying vascular mechanism.

\section{Acknowledgments}

Supported in part by National Institutes of Health/National Eye Institute grants EY011008 (L.M.Z.), EY14267 (L.M.Z.), EY019869 (L.M.Z.), core grant P30EY022589; an unrestricted grant from Research to Prevent Blindness (New York, NY); grants for participants' glaucoma medications from Alcon, Allergan, Pfizer, Merck, and Santen.

\section{Abbreviations and Acronyms}

$\begin{array}{ll}\text {-A } & \text { angiography } \\ \text { CCT } & \text { central corneal thickness } \\ \text { CI } & \text { confidence interval } \\ \text { dB } & \text { decibels } \\ \text { DIGS } & \text { Diagnostic Innovations in Glaucoma Study } \\ \text { IOP } & \text { intraocular pressure } \\ \text { MD } & \text { mean deviation } \\ \text { m } & \text { micrometers }\end{array}$




$\begin{array}{ll}\text { OCT } & \text { optical coherence tomography } \\ \text { POAG } & \text { primary open-angle glaucoma } \\ \text { PSD } & \text { pattern standard deviation } \\ \text { RGC } & \text { retinal ganglion cell } \\ \text { RNFL } & \text { retinal nerve fiber layer } \\ \text { SAP } & \text { standard automated perimetry } \\ \text {-SD } & \text { spectral domain }\end{array}$

\section{REFERENCES}

1. Weinreb RN, Aung T, Medeiros FA. The pathophysiology and treatment of glaucoma: a review. JAMA. 2014; 311(18):1901-11. [PubMed: 24825645]

2. Flammer J. The vascular concept of glaucoma. Surv Ophthalmol. 1994; 38(Suppl):S3-6. [PubMed: 7940146]

3. Weinreb, RN., Harris, A., editors. Ocular Blood Flow in Glaucoma. Kugler Publications; Amsterdam, The Netherlands: 2009.

4. Kornzweig AL, Eliasoph I, Feldstein M. Selective atrophy of the radial peripapillary capillaries in chronic glaucoma. Arch Ophthalmol. 1968; 80(6):696-702. [PubMed: 4177355]

5. Rankin SJ, Drance SM, Buckley AR, Walman BE. Visual field correlations with color Doppler studies in open angle glaucoma. J Glaucoma. 1996; 5(1):15-21. [PubMed: 8795729]

6. Sato EA, Ohtake Y, Shinoda K, et al. Decreased blood flow at neuroretinal rim of optic nerve head corresponds with visual field deficit in eyes with normal tension glaucoma. Graefes Arch Clin Exp Ophthalmol. 2006; 244(7):795-801. [PubMed: 16315043]

7. Hwang JC, Konduru R, Zhang X, et al. Relationship among visual field, blood flow, and neural structure measurements in glaucoma. Invest Ophthalmol Vis Sci. 2012; 53(6):3020-6. [PubMed: 22447865]

8. Harris A, Kagemann L, Cioffi GA. Assessment of human ocular hemodynamics. Surv Ophthalmol. 1998; 42(6):509-33. [PubMed: 9635901]

9. Schuman JS. Measuring Blood Flow: So What? JAMA Ophthalmol. 2015; 133(9):1052-3. [PubMed: 26203625]

10. Bowd C, Zangwill LM, Medeiros FA, et al. Structure-function relationships using confocal scanning laser ophthalmoscopy, optical coherence tomography, and scanning laser polarimetry. Invest Ophthalmol Vis Sci. 2006; 47(7):2889-95. [PubMed: 16799030]

11. Leite MT, Zangwill LM, Weinreb RN, et al. Structure-function relationships using the Cirrus spectral domain optical coherence tomograph and standard automated perimetry. J Glaucoma. 2012; 21(1):49-54. [PubMed: 21952500]

12. Nilforushan N, Nassiri N, Moghimi S, et al. Structure-function relationships between spectraldomain OCT and standard achromatic perimetry. Invest Ophthalmol Vis Sci. 2012; 53(6):2740-8. [PubMed: 22447869]

13. Jia Y, Tan O, Tokayer J, et al. Split-spectrum amplitude-decorrelation angiography with optical coherence tomography. Opt Express. 2012; 20(4):4710-25. [PubMed: 22418228]

14. Spaide RF, Fujimoto JG, Waheed NK. Optical Coherence Tomography Angiography. Retina. 2015; 35(11):2161-2. [PubMed: 26502006]

15. Jia Y, Morrison JC, Tokayer J, et al. Quantitative OCT angiography of optic nerve head blood flow. Biomed Opt Express. 2012; 3(12):3127-37. [PubMed: 23243564]

16. Jia Y, Wei E, Wang X, et al. Optical coherence tomography angiography of optic disc perfusion in glaucoma. Ophthalmology. 2014; 121(7):1322-32. [PubMed: 24629312] 
17. Yu J, Jiang C, Wang X, et al. Macular perfusion in healthy Chinese: an optical coherence tomography angiogram study. Invest Ophthalmol Vis Sci. 2015; 56(5):3212-7. [PubMed: 26024105]

18. Liu L, Jia Y, Takusagawa HL, et al. Optical Coherence Tomography Angiography of the Peripapillary Retina in Glaucoma. JAMA Ophthalmol. 2015; 133(9):1045-52. [PubMed: 26203793]

19. Yarmohammadi A, Zangwill LM, Diniz-Filho A, et al. Optical Coherence Tomography Angiography Vessel Density in Healthy, Glaucoma Suspect, and Glaucoma Eyes. Invest Ophthalmol Vis Sci. 2016; 57(9):OCT451-9. [PubMed: 27409505]

20. Wang X, Jiang C, Ko T, et al. Correlation between optic disc perfusion and glaucomatous severity in patients with open-angle glaucoma: an optical coherence tomography angiography study. Graefes Arch Clin Exp Ophthalmol. 2015; 253(9):1557-64. [PubMed: 26255817]

21. Sample PA, Girkin CA, Zangwill LM, et al. The African Descent and Glaucoma Evaluation Study (ADAGES): design and baseline data. Arch Ophthalmol. 2009; 127(9):1136-45. [PubMed: 19752422]

22. Hodapp, EPRI., Anderson, DR. Clinical decisions in glaucoma. The CV Mosby Co; St Louis, Mo: 1993. p. 52-61.

23. Racette L, Liebmann JM, Girkin CA, et al. African Descent and Glaucoma Evaluation Study (ADAGES): III. Ancestry differences in visual function in healthy eyes. Arch Ophthalmol. 2010; 128(5):551-9. [PubMed: 20457975]

24. Mwanza JC, Warren JL, Hochberg JT, et al. Combining Frequency Doubling Technology Perimetry and Scanning Laser Polarimetry for Glaucoma Detection. J Glaucoma. 2015; 24(8): 561-7. [PubMed: 24777046]

25. Flammer J, Orgul S. Optic nerve blood-flow abnormalities in glaucoma. Prog Retin Eye Res. 1998; 17(2):267-89. [PubMed: 9695795]

26. Weinreb RN, Bartsch DU, Freeman WR. Angiography of the glaucomatous optic nerve head. J Glaucoma. 1994; 3(Suppl 1):S55-60. [PubMed: 19920589]

27. Piltz-seymour JR, Grunwald JE, Hariprasad SM, Dupont J. Optic nerve blood flow is diminished in eyes of primary open-angle glaucoma suspects. Am J Ophthalmol. 2001; 132(1):63-9. [PubMed: 11438055]

28. Grunwald JE, Piltz J, Hariprasad SM, DuPont J. Optic nerve and choroidal circulation in glaucoma. Invest Ophthalmol Vis Sci. 1998; 39(12):2329-36. [PubMed: 9804141]

29. Tobe LA, Harris A, Hussain RM, et al. The role of retrobulbar and retinal circulation on optic nerve head and retinal nerve fibre layer structure in patients with open-angle glaucoma over an 18month period. Br J Ophthalmol. 2015; 99(5):609-12. [PubMed: 25467967]

30. Munguba GC, Galeb S, Liu Y, et al. Nerve fiber layer thinning lags retinal ganglion cell density following crush axonopathy. Invest Ophthalmol Vis Sci. 2014; 55(10):6505-13. [PubMed: 25228542]

31. Chauhan BC, Stevens KT, Levesque JM, et al. Longitudinal in vivo imaging of retinal ganglion cells and retinal thickness changes following optic nerve injury in mice. PLoS One. 2012; 7(6):e40352. [PubMed: 22768284]

32. Leung CK, Chong KK, Chan WM, et al. Comparative study of retinal nerve fiber layer measurement by StratusOCT and GDx VCC, II: structure/function regression analysis in glaucoma. Invest Ophthalmol Vis Sci. 2005; 46(10):3702-11. [PubMed: 16186352]

33. Sehi M, Goharian I, Konduru R, et al. Retinal blood flow in glaucomatous eyes with singlehemifield damage. Ophthalmology. 2014; 121(3):750-8. [PubMed: 24290800]

34. Flammer J, Orgul S, Costa VP, et al. The impact of ocular blood flow in glaucoma. Prog Retin Eye Res. 2002; 21(4):359-93. [PubMed: 12150988]

35. Tokayer J, Jia Y, Dhalla AH, Huang D. Blood flow velocity quantification using split-spectrum amplitude-decorrelation angiography with optical coherence tomography. Biomed Opt Express. 2013; 4(10):1909-24. [PubMed: 24156053]

36. Spaide RF, Fujimoto JG, Waheed NK. Image Artifacts in Optical Coherence Tomography Angiography. Retina. 2015; 35(11):2163-80. [PubMed: 26428607] 
37. Zhi Z, Cepurna WO, Johnson EC, et al. Impact of intraocular pressure on changes of blood flow in the retina, choroid, and optic nerve head in rats investigated by optical microangiography. Biomed Opt Express. 2012; 3(9):2220-33. [PubMed: 23024915]

38. Bengtsson B, Heijl A. A visual field index for calculation of glaucoma rate of progression. Am J Ophthalmol. 2008; 145(2):343-53. [PubMed: 18078852]

39. Rao HL, Senthil S, Choudhari NS, et al. Behavior of visual field index in advanced glaucoma. Invest Ophthalmol Vis Sci. 2013; 54(1):307-12. [PubMed: 23233259]

40. Gardiner SK, Swanson WH, Goren D, et al. Assessment of the reliability of standard automated perimetry in regions of glaucomatous damage. Ophthalmology. 2014; 121(7):1359-69. [PubMed: 24629617]

41. Hood DC, Anderson SC, Wall M, Kardon RH. Structure versus function in glaucoma: an application of a linear model. Invest Ophthalmol Vis Sci. 2007; 48(8):3662-8. [PubMed: 17652736]

42. Hood DC, Kardon RH. A framework for comparing structural and functional measures of glaucomatous damage. Prog Retin Eye Res. 2007; 26(6):688-710. [PubMed: 17889587] 


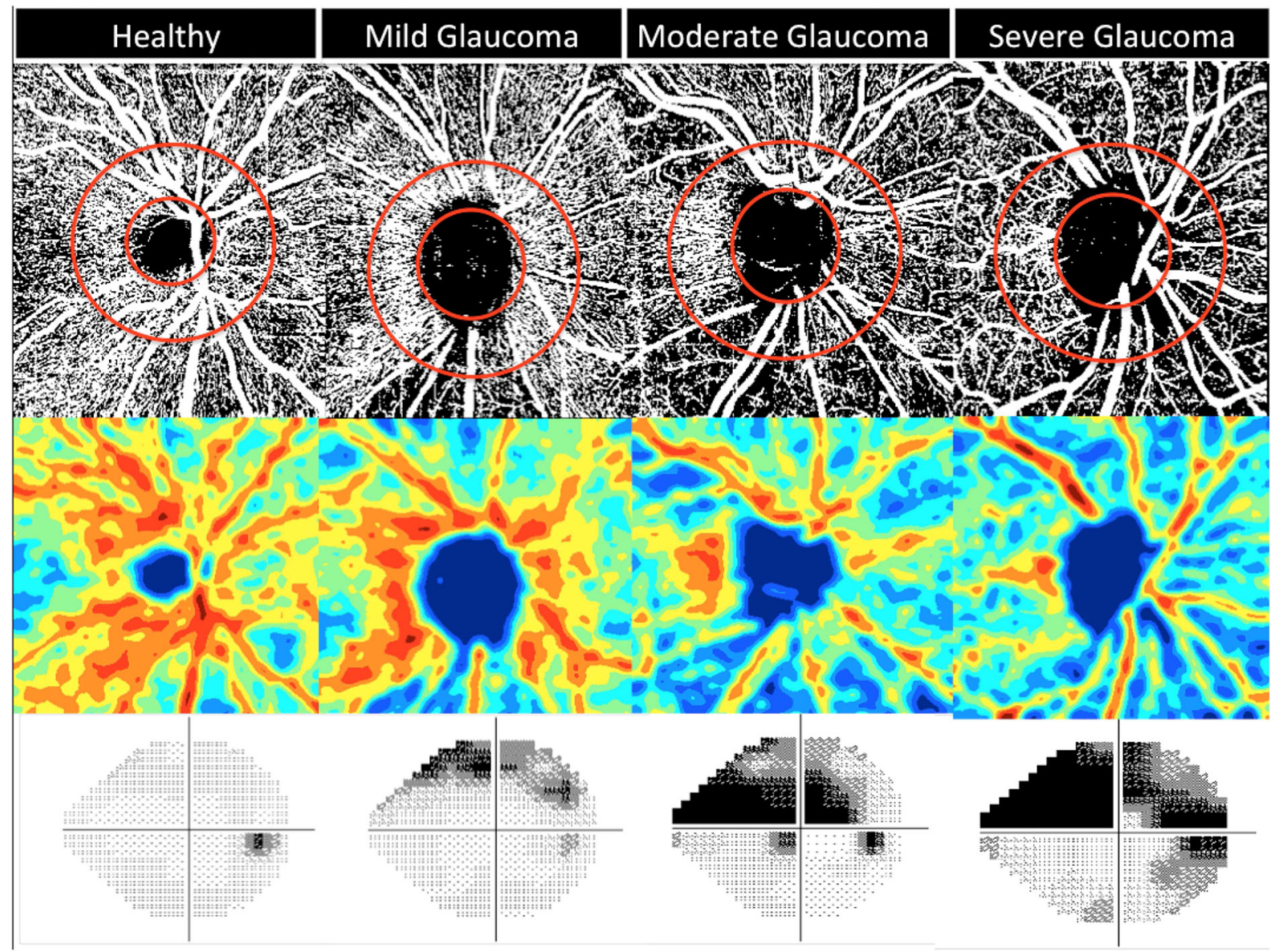

Figure 1.

Vessel Density Map of the Retinal Nerve Fiber Layer showing microvasculature in healthy (most dense), mild, moderate and severe glaucoma (least dense) eye. Top row: Vessel density extracted map with elliptical circumpapillary (cpVD) measurement region defined; Second row: Area vessel density color-coded map; Bottom row: Standard automated perimetry (SAP) visual field results showing corresponding visual field defects. 

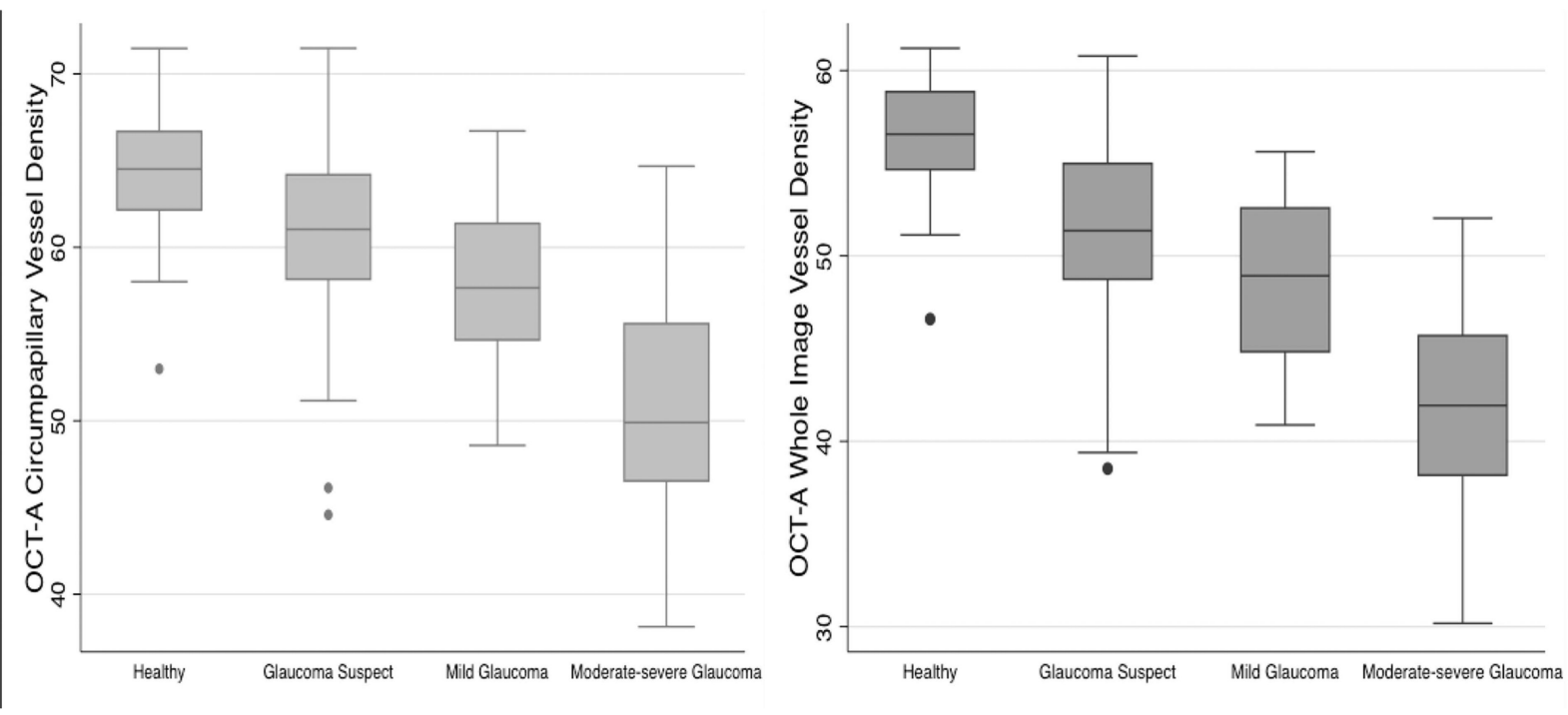

Figure 2.

Boxplots illustrating the distribution of whole image vessel density (left) and circumpapillary vessel density (right) in healthy, glaucoma suspects, mild and moderate to severe glaucoma participants. The medians are represented by horizontal line in the gray box. Error bars represent the interquartile range. 

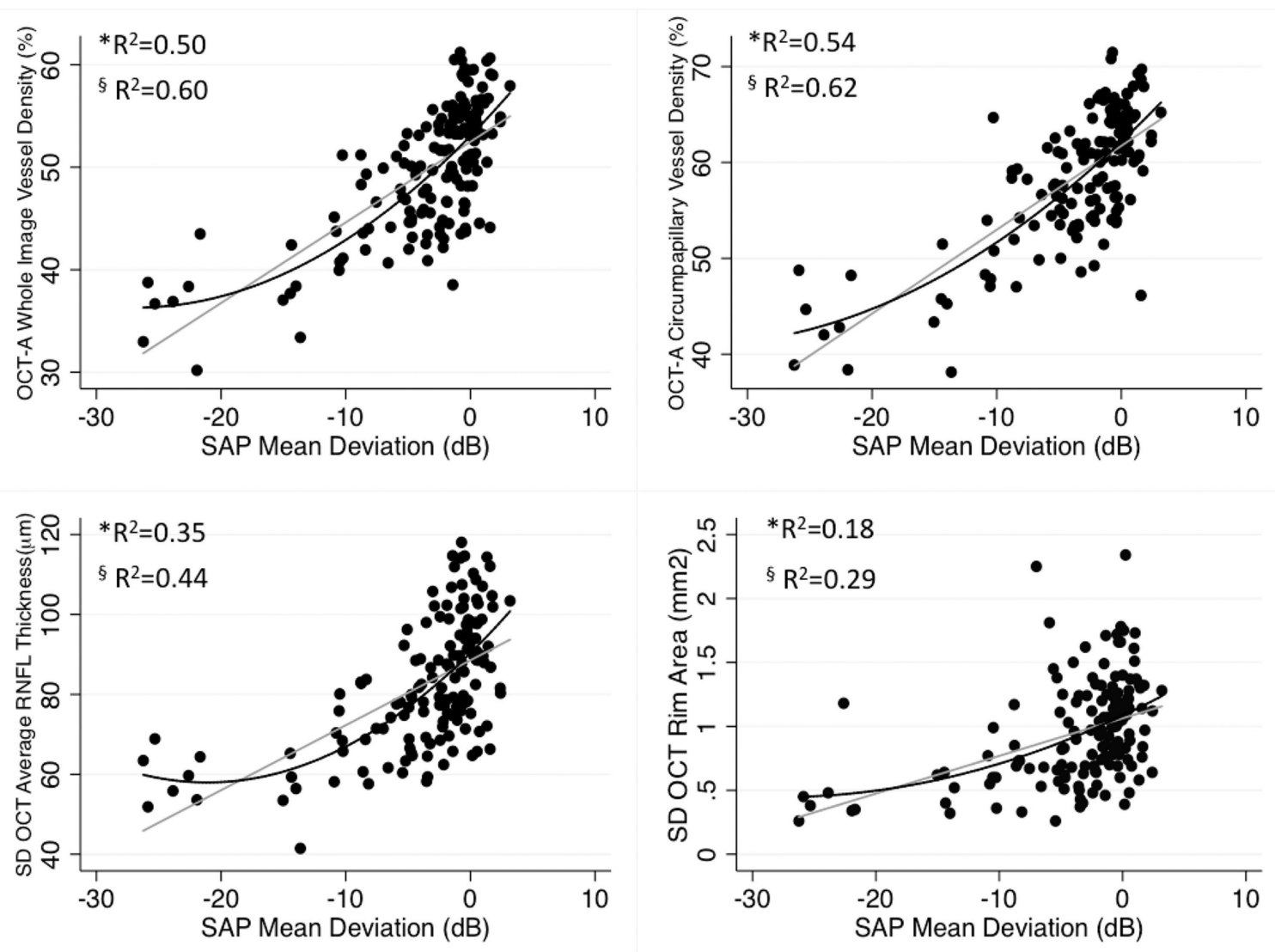

Figure 3.

Scatter plots illustrating the linear (grey line) and curvilinear (quadratic fit: dark lines) correlation between standard automated perimetry (SAP) mean deviation and optical coherence tomography angiography (OCT-A) whole image vessel density, circumpapillary vessel density, Spectral domain (SD) OCT average retinal nerve fiber layer (RNFL) thickness, and rim area measurements. ${ }^{*} \mathrm{R}^{2}$ : Adjusted- $\mathrm{R}^{2}$ from the Linear Regression Model, ${ }^{\S} \mathrm{R}^{2}$ : Adjusted-R ${ }^{2}$ from the Quadratic Regression Model. 

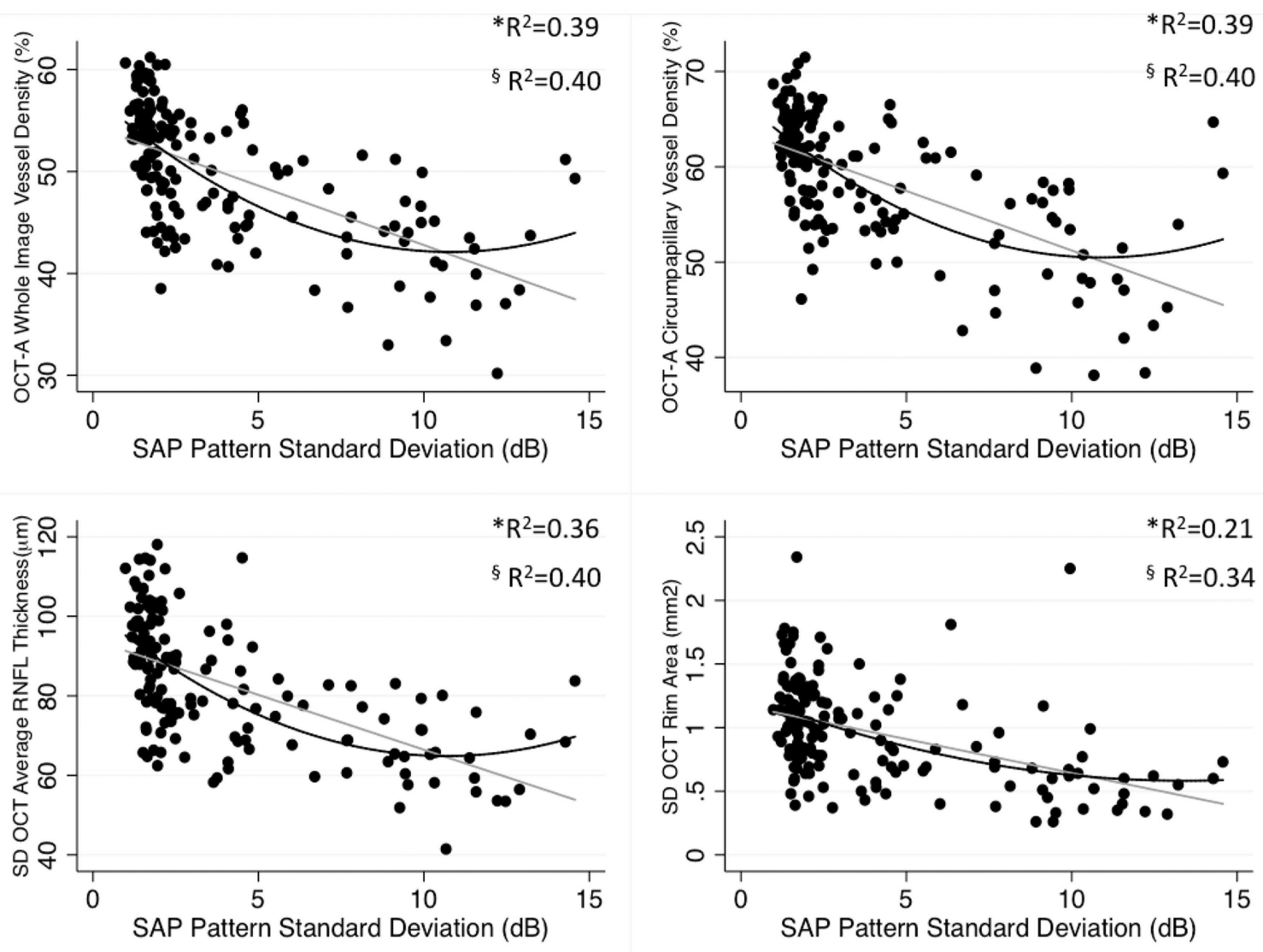

Figure 4.

Scatterplots illustrating the linear (grey line) and curvilinear (quadratic fit: dark lines) correlation between standard automated perimetry (SAP) pattern standard deviation and optical coherence tomography angiography (OCT-A) whole image vessel density, circumpapillary vessel density, Spectral domain (SD) OCT average retinal nerve fiber layer (RNFL) thickness, and rim area measurements. ${ }^{*} \mathrm{R}^{2}$ : Adjusted- $\mathrm{R}^{2}$ from the Linear Regression Model, ${ }^{\S} \mathrm{R}^{2}$ : Adjusted- ${ }^{2}$ from the Quadratic Regression Model. 


\section{Table 1}

Demographics and ocular characteristics of study population.

\begin{tabular}{|c|c|c|c|c|c|}
\hline Variables & Healthy $(n=26)$ & Glaucoma suspect $(\mathrm{n}=52)$ & Mild Glaucoma (n=46) & $\begin{array}{l}\text { Moderate } \\
\text { and Severe } \\
\text { Glaucoma } \\
(\mathbf{n}=28)\end{array}$ & P-value* \\
\hline Age (years) & $55.6(12.5)$ & $68.7(13.1)$ & $72.9(10.7)$ & $75.7(10.7)$ & $<0.001 *^{\star}$ \\
\hline Gender (Male/Female) & $9 / 17$ & $20 / 32$ & $20 / 26$ & $17 / 11$ & 0.19 \\
\hline Ethnicity (AD/ED) & $9 / 17$ & $16 / 36$ & $14 / 32$ & $7 / 21$ & 0.89 \\
\hline \multicolumn{6}{|l|}{ Blood pressure, mm Hg } \\
\hline Systolic & $122.0(16.4)$ & $126.4(19.5)$ & $127.2(13.1)$ & $127.0(15.4)$ & $0.0013^{*}$ \\
\hline Diastolic & $80.8(12.1)$ & $78.8(10.5)$ & $78.6(9.6)$ & $77.8(9.0)$ & 0.82 \\
\hline Mean Blood pressure & $94.5(12.8)$ & $94.7(11.7)$ & $94.8(9.0)$ & $94.2(9.4)$ & 0.61 \\
\hline MOPP, mmHg & $53.0(8.7)$ & $52.1(8.5)$ & $54.4(6.6)$ & $54.6 \mathrm{~b}(7.2)$ & 0.13 \\
\hline Heart rate (bpm) & $71.2(10.8)$ & $68.5(11.7)$ & $66.2(12.1)$ & $64.8(8.2)$ & 0.24 \\
\hline $\begin{array}{l}\text { Self-Reported History of } \\
\text { Diabetes No }(\%)\end{array}$ & 7.0 & 10.0 & 20.0 & 14.0 & $<0.001 *$ \\
\hline $\begin{array}{l}\text { Self-Reported History of } \\
\text { Hypertension No }(\%)\end{array}$ & 31.0 & 54.0 & 61.0 & 68.0 & 0.56 \\
\hline IOP (mm Hg) & $15.1(3.2)$ & $16.5(5.0)$ & $13.2(4.1)$ & $12.3(5.2)$ & $<0.001 * \& \xi$ \\
\hline $\operatorname{CCT}(\mu \mathrm{m})$ & $543.3(41.0)$ & $549.1(39.0)$ & $535.4(37.0)$ & $513.1(36.1)$ & $<0.001 *{ }^{*} \mathcal{S}$ \\
\hline Axial length (mm) & $23.7(1.1)$ & $24.2(1.0)$ & $23.9(1.1)$ & $24.8(1.7)$ & $<0.001 * \square$ \\
\hline Disc Area $\left(\mathrm{mm}^{2}\right)$ & $2.0(0.4)$ & $2.1(0.4)$ & $2.1(0.5)$ & $2.0(0.5)$ & 0.71 \\
\hline $\operatorname{Rim} \operatorname{Area}\left(\mathbf{m m}^{2}\right)$ & $1.3(0.3)$ & $1.0(0.3)$ & $0.9(0.4)$ & $0.7(0.4)$ & $<0.001 *^{\dagger}$ \\
\hline $\begin{array}{l}\text { Average RNFL thickness } \\
(\mu \mathrm{m})\end{array}$ & $99.0(11.4)$ & $87.6(13.0)$ & $78.6(11.6)$ & $65.2(10.4)$ & $<0.001 *^{\dagger}$ \\
\hline SAP Mean Deviation (dB) & $0.4(1.0)$ & $-0.7(1.5)$ & $-3.0(1.8)$ & $-13.6(6.6)$ & $<0.001 * t^{t}$ \\
\hline $\begin{array}{l}\text { SAP Mean Retinal Sensitivity } \\
\text { (dB) }\end{array}$ & $30.6(1.4)$ & $28.6(1.8)$ & $26.1(2.0)$ & $15.5(6.6)$ & $<0.001 * *^{*}$ \\
\hline $\begin{array}{l}\text { SAP Pattern Standard } \\
\text { Deviation }(\mathbf{d B})\end{array}$ & $1.6(0.4)$ & $2.0(0.7)$ & $4.3(2.2)$ & $10.2(2.4)$ & $<0.001 *^{*}$ \\
\hline $\begin{array}{l}\text { OCT-A Whole Image Vessel } \\
\text { Density }(\%)\end{array}$ & $56.7(3.3)$ & $51.6(4.6)$ & $48.3(4.2)$ & $41.7(5.5)$ & $<0.001 *^{\dagger}$ \\
\hline $\begin{array}{l}\text { OCT-A Circumpapillary } \\
\text { Vessel Density (\%) }\end{array}$ & $65.1(3.3)$ & $61.1(4.6)$ & $57.5(4.4)$ & $49.6(6.9)$ & $<0.001 * t$ \\
\hline
\end{tabular}

Statistical significance tested by ANOVA*, corrected with post hoc test $\dagger, \ddagger, \S, \star, \square \star$

Abbreviations: AD: African descent; ED: European descent; SAP: Standard automated perimetry; RNFL: Retinal nerve fiber layer; IOP:

Intraocular pressure; MOPP: Mean ocular perfusion pressure; CCT: Central corneal thickness; OCT-A: Optical coherence tomography angiograph

${ }^{\dagger}$ Significant difference between mean values in all pairwise comparisons.

^Significant difference between mean values in pairwise comparisons of all groups with healthy controls.

${ }^{t}$ Significant difference between mean values in all pairwise comparisons except glaucoma suspects and healthy subjects.

Significant difference between mean values of glaucoma suspects and mild glaucoma patients 
$\xi_{\text {Significant difference between mean values of glaucoma suspects and moderate-severe glaucoma patients }}$

$\square_{\text {Significant difference between mean values of moderate-severe patients with healthy and mild glaucoma patients }}$ 


\section{Table 2}

Age-adjusted Pearson correlation coefficient matrix on vessel density, visual field, and structural variables.

\begin{tabular}{|c|c|c|c|c|c|c|}
\hline Variable & wiVD $(\%)(n=152)$ & $\operatorname{cpVD}(\%)(\mathrm{n}=152)$ & $\begin{array}{l}\text { Average RNFL } \\
\text { Thickness }(\mu \mathrm{m}) \\
(\mathrm{n}=152)\end{array}$ & $\begin{array}{l}\text { SD OCT Rim } \\
\text { Area }\left(\mathbf{m m}^{2}\right) \\
(\mathbf{n}=152)\end{array}$ & $\begin{array}{l}\text { SAP Mean } \\
\text { Deviation (dB) } \\
(n=152)\end{array}$ & $\begin{array}{l}\text { SAP Pattern } \\
\text { Standard } \\
\text { Deviation } \\
(\text { dB })(n=152)\end{array}$ \\
\hline \multicolumn{7}{|l|}{$\begin{array}{l}\text { Whole Image } \\
\text { Vessel Density } \\
(\%)\end{array}$} \\
\hline $\begin{array}{l}\text { Circumpapillary } \\
\text { Vessel Density } \\
(\%)\end{array}$ & ${ }^{*} 0.93(<0.001)$ & & & & & \\
\hline $\begin{array}{l}\text { Average RNFL } \\
\text { Thickness }(\mu \mathrm{m})\end{array}$ & * $0.85(<0.001)$ & ${ }^{*} 0.78(<0.001)$ & & & & \\
\hline $\begin{array}{l}\text { SD OCT Rim } \\
\text { Area }\left(\mathrm{mm}^{2}\right)\end{array}$ & ${ }^{*} 0.63(<0.001)$ & $*^{*} 0.45(<0.001)$ & ${ }^{*} 0.74(<0.001)$ & & & \\
\hline $\begin{array}{l}\text { Visual Field } \\
\text { Mean Deviation } \\
\text { (dB) }\end{array}$ & * $0.71(<0.001)$ & * $0.74(<0.001)$ & ${ }^{*} 0.59(<0.001)$ & ${ }^{*} 0.46(<0.001)$ & & \\
\hline $\begin{array}{l}\text { Visual Field } \\
\text { Pattern } \\
\text { Standard } \\
\text { Deviation (dB) }\end{array}$ & ${ }^{*}-0.63(<0.001)$ & ${ }^{*}-0.63(<0.001)$ & ${ }^{*}-0.60(<0.001)$ & ${ }^{*}-0.48(<0.001)$ & ${ }^{*}-0.79(<0.001)$ & \\
\hline $\begin{array}{l}\text { Visual Field } \\
\text { Mean Sensitivity } \\
\text { (dB) }\end{array}$ & $*^{*} 0.74(<0.001)$ & $*^{*} 0.76(<0.001)$ & $*^{*} .63(<0.001)$ & $*^{*} 0.54(<0.001)$ & ${ }^{*} 1.00(<0.001)$ & $*_{0.81}(<0.001)$ \\
\hline $\begin{array}{l}\text { Visual Field SAP } \\
\text { Mean Sensitivity } \\
\text { (1/lambert) }\end{array}$ & ${ }^{*} 0.74(<0.001)$ & ${ }^{*} 0.72(<0.001)$ & ${ }^{*} 0.71(<0.001)$ & ${ }^{*} 0.65(<0.001)$ & * $0.83(<0.001)$ & ${ }^{*} 0.82(<0.001)$ \\
\hline
\end{tabular}

Abbreviations: SD OCT: spectral domain optical coherence tomography; RNFL: retinal nerve fiber layer; SAP: standard automated perimetry; wiVD: whole image vessel density; cpVD: circumpapillary vessel density.

Pearson's $r$ (P value to test $r=0)$ 


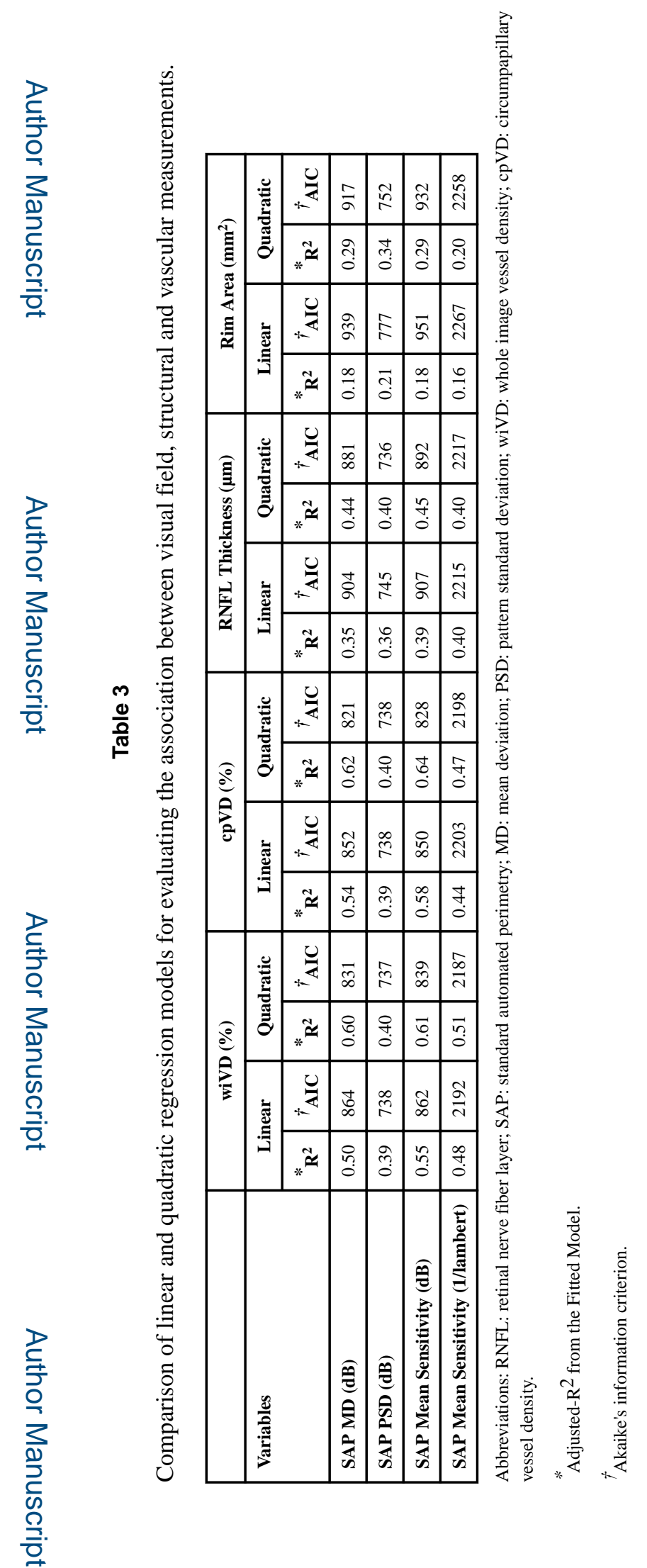

Ophthalmology. Author manuscript; available in PMC 2017 December 01. 
Table 4

Univariate regression analysis for visual field mean deviation.

\begin{tabular}{|c|c|c|c|}
\hline Variables & Coefficient & $\mathbf{R}^{2}$ & P-Value \\
\hline wiVD (per $1 \%$ lower) & -0.64 & 0.501 & $<0.001$ \\
\hline cpVD (per $1 \%$ lower) & -0.62 & 0.540 & $<0.001$ \\
\hline RNFL (per $1 \mu \mathrm{m}$ lower) & -0.22 & 0.353 & $<0.001$ \\
\hline Rim area (per $0.1 \mathrm{~mm}^{2}$ lower) & -0.63 & 0.184 & $<0.001$ \\
\hline Age (per 1 year higher) & -0.12 & 0.080 & $<0.001$ \\
\hline Gender (Female) & 0.91 & 0.006 & 0.340 \\
\hline Race (African descent) & 0.87 & 0.005 & 0.400 \\
\hline IOP (per 1 mm Hg higher) & -0.40 & 0.104 & $<0.001$ \\
\hline CCT (per $10 \mu \mathrm{m}$ lower) & -0.40 & 0.064 & 0.002 \\
\hline Axial length (per 1 mm higher) & -0.90 & 0.040 & 0.022 \\
\hline Mean BP (per $1 \mathrm{~mm} \mathrm{Hg}$ higher) & -0.02 & 0.001 & 0.700 \\
\hline Hypertension (yes) & -0.61 & 0.004 & 0.524 \\
\hline Diabetes (yes) & -1.09 & 0.004 & 0.440 \\
\hline
\end{tabular}

Abbreviations: MD: mean deviation; wiVD: whole image vessel density; cpVD: circumpapillary vessel density; RNFL: retinal nerve fiber layer; IOP: intraocular pressure; CCT: central corneal thickness; BP: blood pressure. 


\section{Table 5}

Multivariate linear regression models to predict visual field mean deviation in association with vessel density, retinal nerve fiber layer thickness measurements.

\begin{tabular}{l|r|r|r}
\hline Variables & Coefficient & 95\% CI & P-Value \\
\hline wiVD (per 1\% lower) & -0.675 & $(-0.87,-0.48)$ & $<0.001$ \\
\hline RNFL (per 1 $\mu$ m lower) & -0.003 & $(-0.08,0.08)$ & $<0.938$ \\
\hline Age (per 1 year higher) & -0.065 & $(-0.13,-0.01)$ & 0.033 \\
\hline IOP (per 1 mm Hg higher) & -0.212 & $(-0.36,-0.07)$ & 0.005 \\
\hline CCT (per 10 $\mu \mathrm{m}$ lower) & -0.004 & $(-0.02,0.01)$ & 0.638 \\
\hline Axial length (per 1 mm higher) & -0.136 & $(-0.73,0.45)$ & 0.649 \\
\hline
\end{tabular}

Abbreviations: CI: confidence interval; wiVD: whole image vessel density; RNFL: retinal nerve fiber layer; IOP: intraocular pressure; CCT: central corneal thickness. 


\section{Table 6}

Multivariate linear regression models to predict visual field mean deviation in association with vessel density, rim area measurements.

\begin{tabular}{l|r|r|r}
\hline Variables & Coefficient & 95\% CI & P-Value \\
\hline wiVD (per 1\% lower) & -0.700 & $(-0.85,-0.55)$ & $<0.001$ \\
\hline Rim area (per 0.1 mm ${ }^{2}$ lower) & -0.818 & $(-1.39,3.02)$ & 0.464 \\
\hline Age (per 1 year higher) & -0.069 & $(-0.13,-0.01)$ & 0.025 \\
\hline IOP (per 1 mm Hg higher) & -0.213 & $(-0.36,-0.07)$ & 0.005 \\
\hline CCT (per 10 $\mu \mathrm{m}$ lower) & -0.005 & $(-0.02,0.01)$ & 0.576 \\
\hline Axial length (per 1 mm higher) & -0.091 & $(-0.68,0.50)$ & 0.761 \\
\hline
\end{tabular}

Abbreviations: CI: confidence interval; wiVD: whole image vessel density; IOP: intraocular pressure; CCT: central corneal thickness. 